American Journal of Environmental Sciences 8 (3): 195-201, 2012

ISSN 1553-345X

(C) 2012 Science Publications

\title{
Overview of Membrane Processes for the Recovery of Polyphenols from Olive Mill Wastewater
}

\author{
Ompe Aime Mudimu, Martin Peters, Florian Brauner and Gerd Braun \\ Cologne University of Applied Sciences, Cologne, Germany
}

\begin{abstract}
Problem statement: Olive Mill Wastewater (OMW) is an ambivalent by-product of the olive oil production, which appears in huge amounts every year after the olive-harvest in the production countries, mainly in the Mediterranean region. OMW is characterized by a high organic load and i.a. contains considerable amounts of phytotoxical polyphenols, which cause important environmental problems. Approach: Due to their antioxidant properties the use of these polyphenols is also popular in several industry branches, which results in high sale values for this group of chemicals. The removal of polyphenols from biological wastewaters like OMW does not only reduce the pollutant load but also shows great potential for a beneficial recovery of these antioxidants. This is the reason why a growing number of studies deal with a combined wastewater treatment, which, besides water purification, also regards the ability of recovering polyphenols. This article is an overview of reports concerning polyphenol recovery from OMW via membrane technologies. Results: Patents and studies, which appeared in literature, are reviewed in order to identify the potential of membranes as well as making comparisons possible. Some pretreatments, feasible for membrane processes, are covered. Depending on the initial wastewater and its polyphenol content the concentration in the obtained solution ranges from 0.5-19.3 $\mathrm{g} \mathrm{L}^{-1}$ polyphenols. An example mentioned in WO2005/123603 even obtains a concentration of $30 \mathrm{~g} \mathrm{~L}^{-1}$. Polyphenols such as hydroxytyrosol, protocatechuic acid, tyrosol, caffeic acid and oleuropein were found in this concentrate and make it suitable for the use in industry. Conclusion: The membrane processes in sequential design in particular show good results and offer an alternative to other OMW treatments especially in terms of polyphenol recovery.
\end{abstract}

Key words: Olive mill, membrane processes, polyphenol recovery, mill wastewater, polyphenol content, protocatechuic acid, polyphenol recovery

\section{INTRODUCTION}

The production of olive oil, extracted from the olive fruit, involves the generation of several wastes of solid, liquid and semi-solid type. Especially the liquid fraction, called Olive Mill Wastewater (OMW), causes adverse effects on the environment, due to its phytotoxical compounds (mainly polyphenols) and because it occurs in great quantities and in a short period of time after the oliveharvest in November (Torrecilla, 2010).

A large number of small olive mills, spread across the Mediterranean region, produce about $97 \%$ of the total olive oil production worldwide. A lot of these small enterprises, deterred by the high investment costs of purification plants, still spread their wastewater on the fields or discharge it into open waters, despite its environmental impact (Paraskeva and Diamadopoulos, 2006).

However, there have been efforts to solve these environmental problems in the past few decades
(Niaounakis and Halvadakis, 2006) and scores of references and patents concerning various wastewater treatments, including physical, physicochemical, thermal and biological processes, have been published worldwide.

Nevertheless, none of them has been widely accepted so far. Paraskeva and Diamadopoulos (2006), who reviewed several technologies for olive mill wastewater purification, came to the conclusion that "an environmentally safe, cost-effective solution to OMW treatment has yet to be found" (Kapellakis et al., 2006).

Apart from purifying the wastewater, there have been attempts to make use of the polyphenols contained in the OMW. While these components are the main reason for the wastewater's phytotoxicity, they also show useful characteristics as antioxidants (Marco et al., 2007). Hydroxytyrosol and catechol for instance are popular due to their protective action on human health and counter effects in pathological processes such as cancer and atherosclerosis (Visioli et al., 1999). Other

Corresponding Author: Ompe Aime Mudimu, Cologne University of Applied Sciences, Cologne, Germany 
phenols can be found e.g., in varnishes, pesticides and in the production processes of drugs, cosmetics and synthetics. The use in different branches of industry cause high sale values (Obied et al., 2005) and make the recovery of these components from OMW attractive.

Membrane separation is one technique, which besides purifying the wastewater, is also able to recover polyphenols from OMW. The small area-requirement and low energy-consumption of this operation make it popular in water treatment and feasible for OMW treatment as well. The specific separation limits of sequent membrane processes allow adjusting this treatment with regard to the particular wastewater, whose characteristics vary with olive origin, extraction method and other factors.

Membranes separate different components, e.g., in a solution by means of their unequal particle sizes (Microfiltration, Ultrafiltration and Nanofiltration) or differences in other characteristics, which affect their permeability, such as molecular weight (Reverse Osmosis) or state of aggregation (Membrane Distillation and Osmotic Distillation) (Crittenden, 2005).

The wastewater treatment via membrane techniques obtains two phases: One which passes through the membrane (permeate) and one which remains on the feed side of the membrane (concentrate or retentate). In case of OMW that means that larger parts such as suspended solids (and therewith great amounts of organic carbon) can be separated from the wastewater by Microfiltration (MF) or Ultrafiltration (UF) and then can be found in the membrane's retentate. Polyphenols have molecular weights (MW) ranging from 138,164 $\mathrm{g} \mathrm{mL}^{-1}$ (tyrosol) to $540,514 \mathrm{~g} \mathrm{~m}$ $\mathrm{L}^{-1} \quad$ (oleuropein) and can pass these types of membranes more or less unhindered. Then, after separating the phenolic compounds from the other organic constituent parts, Nanofiltration (NF) and Reverse Osmosis (RO) can be used for further treatment. Due to its smaller molecular weight cut-off, these membranes are able to retain polyphenols in great amounts and in that way produce a concentrated solution of polyphenols. The permeate, which is cleaned of other constituent parts, then is a harmless water and can be used for internal processes in the olive mill or for instance for irrigation. The membrane processes, presented in this study, partially have been used already for the OMW treatment or even have been patent-registered. Others just have been tested in laboratory scale only. Not all studies give numerical results and the variable composition of OMW samples complicate the comparison of given results even more. Still, the aim of this study is to provide an overview of membrane processes, which might be useable for the treatment of OMW including polyphenol recovery and to allow comparisons between diverse plants and equipment. Takac and Karakaya (2009) argue that membranes in a sequential design seem to be the "future direction" of OMW processing and recovery of antioxidants (Takac and Karakaya, 2009).

COD reduction: In order to obtain a polyphenol concentrate, the wastewater's pollution load has to be minimized in the first instance. Great parts of the organic substances are included in the suspended solids. That is why it is reasonable to achieve a reduction of the Chemical Oxygen Demand (COD) by retaining the suspended solids for instance via micro- or ultrafiltration. These procedures can be supported optionally by a prior enzymatic treatment. The following studies gave examples for COD reducing pretreatments.

In the eldest publication which is treated in this study Canepa et al. (1988) described a preliminary ultra-filtration of the raw wastewater to reduce the COD. The UF pilot unit was made of four multiple modules in series with three tubular membranes each for a total surface area of $2 \mathrm{~m}^{2}$ and was equipped with polysulfone membranes with a nominal cut-off of 20 $\mathrm{kDa}$. For the complete procedure, the temperature was kept constant at $20^{\circ} \mathrm{C}$, operating pressure at $3 \times 1.02$ bar and flow rate at $3 \mathrm{~m} \mathrm{~s}^{-1}$. This UF step removed parts of the organic content (suspended solids) and showed a total COD reduction of about $63 \%$.

In patent WO2005/123603 from 2005 Pizzichini and Russo (2005) used a combination of centrifugation, MF and UF to obtain a reduction in COD which was supported by a prior $\mathrm{pH}$-adjustment and an enzymatic hydrolysis. The $\mathrm{pH}$-adjustment to a value within 3 and 4.5 avoided the oxidation of the polyphenolic compounds, favoured the transformation of oleuropein into hydroxytyrosol and moreover created the optimal conditions for the following enzymatic treatment. The enzymatic hydrolysis degraded and separated organic compounds such as cellulose out from the wastewater to allow a further separation via centrifugation and to reduce the clogging effect on the membranes due to solid components. The liquid stream from the centrifugation step was then subjected to MF, which was carried out with ceramic membranes with separation limits ranging from $0.1-1.4 \mu \mathrm{m}$ and a surface area of $0.35 \mathrm{~m}^{2}$ per ceramic block. Then the MF permeate was proceeded by an UF step, which used spiral-wound polymeric membranes with cut-offs ranging between 1 and $20 \mathrm{kDa}$.

Two years later, in 2007, Russo (2007) described an experimental study, which investigated fouling effects and the removal efficiencies of several MF and UF membranes considering their potential to produce a 
Am. J. Environ. Sci., 8 (3): 195-201, 2012

polyphenol concentrate. Membranes with differential cutoffs and made of diverse materials were tested with fixed process parameters and without preliminary centrifugation but with a prior $\mathrm{pH}$-adjustment to $\mathrm{pH}=$ 3.5 to prevent phenols oxidation. The MF process was declared as the "critical section of the process" due to fouling effects. Thus three different membranes were tested for this process, comparing productivity and fouling effects (Table 1).

The polymeric $500 \mathrm{kDa}$ membrane showed a quick decreasing of the permeate flux of more than $70 \%$ due to fouling. The ceramic membranes had smaller fouling indexes than the polymeric one and were both of the same order of magnitude. The smallest fouling index was measured for the $0.45 \mu \mathrm{m}$ membrane. Although the fouling processes were still high, this membrane was pointed out to be the preferential one for the MF operation. The concentration of free low molecular weight polyphenols was $55.38 \mathrm{ppm}$ in the untreated OMW in this study and grew to a value of $349.18 \mathrm{ppm}$ in the MF $0.45 \mu \mathrm{m}$ permeate. With a concentration of $266.679 \mathrm{ppm}$, hydroxytyrosol accounts for $76 \%$ thereof. Other polyphenols (such as oleuropein, tyrosol, protocatechuic acid and caffeic acid) had concentrations of about $20 \mathrm{ppm}$. Alternatively to the MF process, four UF membranes were analyzed treating the same feed solution: Three polymeric membranes, supplied by GE Osmonics with cut-offs of 80, 20 and $6 \mathrm{kDa}$ and then a ceramic membrane, supplied by TAMI (cut-off $=1$ $\mathrm{kDa}$ ), which further treated the permeate, produced by the kDa UF membrane (Table 2).

The combination of UF $6 \mathrm{kDa}$ and UF $1 \mathrm{kDa}$ was pointed out to be the most effective treatment in this process step. Amongst others, UF $6 \mathrm{kDa}$ on MF $0.45 \mu \mathrm{m}$ permeate showed a rejection of about $45 \%$ of free polyphenols. The rejection of oleuropein reached $75 \%$ and hydroxytyrosol of about $45 \%$ as well. Then again UF $1 \mathrm{kDa}$ on UF $6 \mathrm{kDa}$ permeate reached rejections of free polyphenols and hydroxytyrosol of $32,31 \%$ respectively. Then UF $6 \mathrm{kDa}$ permeate and UF $1 \mathrm{kDa}$ permeate showed a concentration of free low MW polyphenols of $230.413 \mathrm{ppm}$ and $170.053 \mathrm{ppm}$, respectively.

Paraskeva (2007) described a study, which used UF for the reduction of organic substances (COD). The OMW samples, prefiltered with a polypropylene screen $(80 \mu \mathrm{m})$ to remove suspended solids, were poured onto an UF membrane made of zirconium oxide with pore sizes of $100 \mathrm{~nm}$ and a surface area of $0.24 \mathrm{~m}^{2}$. This UF step achieved reductions of 97\% Total Suspended Solids (TSS), 17\% Total Organic Carbon (TOC), 21\% COD and $34 \%$ phenols using a pressure of 1.75 bar. While great parts of the organic pollution could be retained, the main part of the phenolic compounds passed the UF membrane and were available for further operation steps.
Table 1: MF membranes tested by Russo

\begin{tabular}{llll}
\hline MF Membrane & Material & Cut-off & Area $\left(\mathrm{m}^{2}\right)$ \\
\hline Ceramic Tami & Zirconium oxide & $0.80 \mu \mathrm{m}$ & 0.35 \\
Ceramic Tami & Zirconium oxide & $0.45 \mu \mathrm{m}$ & 0.35 \\
Polymeric Nadir & Spiral-wound PE & $500 \mathrm{kDa}$ & 3.80 \\
\hline
\end{tabular}

(Data: Russo, 2007, p. 242)

Table 2: UF membranes tested by Russo

\begin{tabular}{llll}
\hline UF Membrane & Material & Cut-off & Area $\left(\mathrm{m}^{2}\right)$ \\
\hline Polymeric GE Osmonics & Spiral-wound PS & $80 \mathrm{kDa}$ & 5.00 \\
Polymeric GE Osmonics & Spiral-wound PS & $20 \mathrm{kDa}$ & 5.00 \\
Polymeric GE Osmonics & Spiral-wound PS & $6 \mathrm{kDa}$ & 8.36 \\
Ceramic Tami & Zirconium oxide & $1 \mathrm{kDa}$ & 0.35 \\
\hline PS = Polysulfone; PES = Polyethersulfone (Data: Russo, 2007, p. 242)
\end{tabular}

A combination of UF and NF processes for OMW treatment was investigated by Stoller (2008). First the critical fluxes were determined and the appropriate operating conditions for the UF and the NF process were calculated. In the UF step the OMW, pretreated by an aerobic biological operation, was filtered using the GE Osmonics GM4040F membrane (composite thinfilm Desal-5). The temperature in the feed vessel was maintained with a heat exchanger between $16-18^{\circ} \mathrm{C}$. The permeate flux was calculated as $6.25 \mathrm{l} /\left(\mathrm{h} \mathrm{m}^{2}\right)$, the pressure values were adjusted (2.2-2.5 bar) to keep this flow rate constant. The NF process was then fed with the UF permeate using the GE Osmonics DK4040F membrane (also composite thin-film Desal-5). The temperature in the feed vessel was again kept between $16-18^{\circ} \mathrm{C}$. For the NF process, Stoller calculated a permeate flux of $4.21 \mathrm{l} /\left(\mathrm{m}^{2} \mathrm{~h}\right)$ to be effective. By adjusting the operating pressure, this value was maintained constant. The UF step showed rejection values of $50 \%$ for COD and $47 \%$ for polyphenols and delivered a residue with a content of $15.65 \mathrm{mg} \mathrm{L}^{-1}$ polyphenols (initial value was $3.1 \mathrm{mg} \mathrm{L}^{-1}$ ). The $\mathrm{NF}$ observed a rejection value of 77 for COD and $97 \%$ for polyphenols. The NF residue contained a polyphenolic content of $16.0 \mathrm{mg} \mathrm{L}^{-1}$ (feed concentration was $1.64 \mathrm{mg}$ $\mathrm{L}^{-1}$ ). As the UF step already severely reduced the amount of contained polyphenols (only $53 \%$ pass the membrane) the concentration factor for further treatments is limited. It was noted that the NF permeate was a harmless water, which could be used for irrigation or be discharged in the municipal sewer system.

In patent No. US2009/23815 Villanova et al. (2008) focused the preparation of tyrosol and hydroxytyrosol from OMW. The prior reduction of the pollution load was achieved by two sequent pretreatment units and another two modules equipped with UF membranes in the final unit. The first pretreatment unit was equipped with seven rough filtration modules ranging from 4.76$0.033 \mathrm{~mm}$. The second consisted of two $0.24 \mathrm{~m}^{2}$ modules, which were equipped each with a $120 \mathrm{kDa} \mathrm{MF}$ 
membrane. The UF membranes in the final unit had cutoffs of about 120-20 kDa and 20-1 kDa and areas both of $1.6 \mathrm{~m}^{2}$. COD reduction values were not specified.

In an integrated membrane system for the polyphenol recovery Garcia-Castello et al. (2010) used MF and NF steps as pretreatments for reduction of the organic pollution. For the MF a laboratory pilot unit (supplied by Verind SpA, Milano, Italy) was equipped with a tubular membrane made of $\mathrm{Al} 2 \mathrm{O} 3$ (pore sizes $200 \mathrm{~nm}$, surface area $48 \mathrm{~cm}^{2}$ ) supplied by Inopor $\mathrm{GmbH}$ (Hermsdorf, Germany). Operating with a transmembrane pressure (TMP) of $0.72+/-1$ bar and a temperature of $22+/-0.01^{\circ} \mathrm{C}$, the feed flow rate achieved $760 \mathrm{l} / \mathrm{h}$. Starting with a feed solution containing $20.19 \mathrm{~g} \mathrm{~L}^{-1}$ TOC, the MF process reduced the TOC to a value of $15.01 \mathrm{~g} \mathrm{~L}^{-1}$. The amount of suspended solids was even reduced from $17.6 \mathrm{~g} \mathrm{~kg} \mathrm{~L}^{-1}$ $1.6 \mathrm{~g} \mathrm{~kg} \mathrm{~L}^{-1}$. The NF process was performed with a laboratory plant supplied by Matrix esalination Inc. (USA), equipped with a Nadir N30F spiral-wound membrane made of polyethersulfone (surface area of $1.6 \mathrm{~m}^{2}$, MWCO $578 \mathrm{Da}$ ), supplied by Microdyn-Nadir $\mathrm{GmbH}$ (Venlo, Netherlands). Operating temperature was $20^{\circ} \mathrm{C}$ and TMP about 8 bar. The content of TOC was further reduced by the NF step and reached a value of only $5.57 \mathrm{~g} \mathrm{~L}^{-1}$.

Recently Servili et al. (2011) described a threephase membrane system in industrial scale which used a consecutive filtration with MF and UF in combination with an enzymatic treatment to reduce the OMW pollution. Heat exchangers kept a constant temperature of $20^{\circ} \mathrm{C}$ for the whole treatment and a nitrogenatmosphere in the storage vessels reduced the content of oxygen. The MF process was performed by a tubular Poly-Propylene (PP) membrane with a cut-off ranging from $0.1-0.3 \mu \mathrm{m}$ and a total surface area of $8 \mathrm{~m}^{2}$. The UF process consisted of two spiral membranes made of polyamide and traces of polysulfone and treated the MF permeate. The MWCO of this membrane was stated as $7 \mathrm{kDa}$ and the total surface area was $16 \mathrm{~m}^{2}$. The entire process which included a final RO step for concentration, reduced the initial COD of $12.9 \mathrm{~g} \mathrm{~L}^{-1}$ with a drawdown of $98 \%$ to a COD value of $2.47 \mathrm{~g} \mathrm{~L}^{-1}$. Values for the separate membrane steps were not given.

Recovery of phenolic compounds: After separating the organic load from the wastewater, the phenolic compounds with less molecular weights can be recovered and concentrated by use of NF and RO because their cut-offs are even smaller. Most studies which were mentioned in the previous chapter also covered several options for the recovery of polyphenols via membrane technologies.
After the pretreatment for COD reduction Canepa et al. (1988) described a combination of RO and adsorbing on porous polymers for the phenol recovery. The adsorbing step showed good retention qualities for polyphenols, but is not noted in detail at this point. The focus of attention is on the reverse osmosis operation, which used the resins effluent as feed solution. The RO step consisted of two units working in series and equipped with spiral-wound modules: (1) with a nominal rejection of $97 \%$ to $\mathrm{NaCl}$, working with a TMP of $0.4 \mathrm{bar}$ and (2) with a nominal rejection of $95 \%$ to $\mathrm{NaCl}$ and anoperating pressure of 1.02 bar. Both membranes were made of polypiperazine amide and worked at a constant temperature of $20^{\circ} \mathrm{C}$. This RO process showed a concentration of phenolic compounds from index 86 (RO feed) to 490 (RO retentate). Besides a further COD reduction of $93 \%$ and TSS (total suspended solids) reduction of $99 \%$ was shown.

The description in Patent No. WO2005/123603 (Pizzichini and Russo, 2005), which used MF and UF for COD reduction, arranged the phenol recovery by subsequent NF and RO. The NF worked with spiralshaped polymeric membranes having cut-offs ranging from 150-250 Da. The NF permeate, which contained great amounts of polyphenolic compounds, was then given to the final RO operation, which was characterized by spiral-shaped polymeric membranes made of composite polyamide having high saline rejections. This final operation yielded a concentrate rich in polyphenolic compounds and a permeate consisting of purified water. The entire process can be supported by Diafiltration to improve the benefit. Examples with this operation set-up, which was fed with $2001 \mathrm{OMW}$, coming from a three-phase system, obtained about 121 of concentrate, which contained around $30 \mathrm{~g} \mathrm{~L}^{-1}$ total polyphenols.

The experimental study of Russo (2007) concerning removal efficiencies of several MF and UF membranes concentrated the recovered polyphenols by using a final RO consisting of a polymeric Hydronautics membrane (composite polyamide) with an area of $7 \mathrm{~m}^{2}$. With a concentration factor of 2.5 , the RO membrane produced a retentate containing 464.870 ppm free low MW polyphenols composed of 390.449 ppm hydroxytyrosol, $27.400 \mathrm{ppm}$ tyrosol, $14.968 \mathrm{ppm}$ oleuropein, 14.329 ppmcaffeic acid and 17.724 ppm protocatechuic acid. Because of preliminary treatments, fouling problems were minimized and the membrane regeneration was obtained by washing with water.

The concentration efficiencies of NF and RO were compared in Paraskeva et al. (2007) after an UF process. Therefore NF and RO tests both used spiralwound polymeric membranes (area of $2.5 \mathrm{~m}^{2}$ ) with a Molecular Weight Cut-Off (MWCO) of 200 and $100 \mathrm{Da}$, respectively. 
Am. J. Environ. Sci., 8 (3): 195-201, 2012

Table 3: Polyphenol concentration found in several process steps in Garcia-Castello et al. (2010) (data in ppm)

\begin{tabular}{|c|c|c|c|c|c|c|}
\hline & \multirow[b]{2}{*}{ Initial OMW } & \multicolumn{2}{|l|}{ MF } & \multicolumn{2}{|l|}{$\mathrm{MF}$} & \multirow{2}{*}{$\begin{array}{l}\text { OD } \\
\text { Concentrate }\end{array}$} \\
\hline & & Permeate & Concentrate & Permeate & Concentrate & \\
\hline Free low MW polyphenols & 211.80 & 170.54 & 311.87 & 160.32 & 220.10 & 493.01 \\
\hline Hydroxytyrosol & 108.00 & 88.71 & 196.31 & 85.00 & 118.00 & 276.00 \\
\hline Protocatechuic acid & 16.50 & 15.31 & 19.00 & 12.13 & 14.80 & 29.00 \\
\hline Tyrosol & 15.00 & 11.28 & 25.56 & 9.24 & 15.00 & 53.50 \\
\hline Caffeic acid & 9.95 & 8.70 & 9.00 & 8.55 & 9.95 & 49.00 \\
\hline p-Cumaric acid & 8.35 & 7.54 & 12.00 & 6.70 & 8.35 & 20.51 \\
\hline Oleuropein & 54.00 & 39.00 & 50.00 & 38.70 & 54.00 & 65.00 \\
\hline
\end{tabular}

(Data: Garcia-Castello et al., 2010, p. 3888)

Operating pressure was between 10-30 bar for the NF between 30-40 bar for RO. Feeding with the UF permeate, the NF step showed reduction efficiencies of 99 TSS, 97 TOC, 97 COD and 98\% phenols. While most phenols could pass the prior UF membrane, they were retained by the NF membrane. The NF retentate contained a concentration of $9.962 \mathrm{~g} \mathrm{~L}^{-1}$ of phenols (with respect to a feed concentration of $0.725 \mathrm{~g} \mathrm{~L}^{-1}$ ). Alternatively operating the UF permeate with the RO membrane obtained similar reduction results. The content of polyphenols in the RO concentrate was then determined as $6.782 \mathrm{~g} \mathrm{~L}^{-1}$ (feed had $1.018 \mathrm{~g} \mathrm{~L}^{-1}$ ).

Nunes et al. (2007) described the production of a hydroxytyrosol concentrate by use of nanofiltration and reverse osmosis procedures in patent number WO2007/013032. An aqueous extract (olive cake plus water) was given to a NF membrane Desal DK type (supplied by GE Osmonics) with a MWCO of $250 \mathrm{Da}$ at a pressure difference of $10 \mathrm{bar}$. This process yielded a recovery of hydroxytyrosol of about $70 \%$. The following RO process further concentrated the obtained NF retentate using a Dow Filmtec SW 30 membrane (from Dow) operating at 25 bar.

The use of Direct Contact Membrane Distillation (DCMD) for the concentration of phenolic compounds was investigated by El-Abbassi et al. (2009) taking OMW samples (press) from the region of Marrakech (Morocco). Two commercial flat-sheet hydrophobic membranes were compared to each other:

- Gelman TF200 made of PTFE

- (Polytetrafluoroethylene), mean pore size: $198.96 \mathrm{~nm}$

- Millipore GVHP made of PVDF

- Polyvinylidene fluoride), mean pore size: $283.15 \mathrm{~nm}$

With a concentration factor of 1.72 , the TF200 membrane obtained a retentate, which had a polyphenol content of about $6.88 \mathrm{~g} \mathrm{~L}^{-1}$ (initial value was $4 \mathrm{~g} \mathrm{~L}^{-1}$ ). However the Millipore GVHP membrane showed a concentration factor of 1.4 and thus a polyphenol content of only $5.6 \mathrm{~g} \mathrm{~L}^{-1}$ was reached. Furthermore it was found that the TF200 showed a higher permeate flux $\left(7.68 \pm 0.22 \mathrm{l} /\left(\mathrm{h} \mathrm{m}^{2}\right)\right)$ than the GVHP membrane.
Villanova (2008) described one NF and one RO module for the concentration of the pretreated OMW. Placed in the third unit after two UF modules, the NF membrane (area of $2.5 \mathrm{~m}^{2}, 1000-350 \mathrm{kDa}$ cut-off) and following RO membrane (area of $2.5 \mathrm{~m}^{2}, 350 \mathrm{kDa}$ cutoff) yielded concentrations of hydroxytyrosol of more than $1 \mathrm{~g} \mathrm{~L}^{-1}$ (initial OMW had $0.26 \mathrm{~g} \mathrm{~L}^{-1}$ ) and tyrosol of morethan $0.6 \mathrm{~g} \mathrm{~L}^{-1}$ (initial was $0.08 \mathrm{~g} \mathrm{~L}^{-1}$ ).

The integrated membrane system proposed by Garcia-Castello et al. (2010) investigated the concentration abilities of Osmotic Distillation (OD) and Vacuum Membrane Distillation (VMD) after a NF process. The OD was performed with a compact plant supplied by Celgard LLC (Charlotte, USA) with a Liqui-Cel ${ }^{\circledR}$ Extra-Flow $2.5 \times 8$ ' membrane module (also Celgard LLC) made of polyethylene with a surface area of $1.4 \mathrm{~m}^{2}$ (effective area/volume $29.3 \mathrm{~cm}^{2} / \mathrm{cm}^{3}$ ), containing microporous polypropylene hollow-fibers (mean pore size about $30 \mathrm{~nm}$ ). With an input content of polyphenols of $160.32 \mathrm{ppm}$ (NF permeate), the OD process achieved a polyphenolic concentration of $493.01 \mathrm{ppm}$ in OD retentate. To compare the evaporation fluxes of the two concentration systems $\mathrm{OD}$ and VMD, the NF permeate was also used in a VMD process consisting of a laboratory set-up with a flat membrane module (membrane area of $55 \mathrm{~cm}^{2}$ ) equipped with a flat-sheet hydrophobic and microporous membrane made of Polyvinylidene Fluoride (PVDF). The membrane was $200 \mu \mathrm{m}$ thick and its pore size was $0.2 \mu \mathrm{m}$. In swelling experiments, the PVDF membrane was compared to a Polypropylene (PP) membrane and showed the lowest swelling degree. That underlined its selection for use in the VMD process. In comparison with OD, the VMD process had a higher energy consumption, which is why the OD process was declared as preferred technology in this study, although the transmembrane fluxes were higher in the VMD process. The whole membrane system delivered a liquid concentrate containing about $0.5 \mathrm{~g} \mathrm{l}^{-1}$ free low MW polyphenols. As was already mentioned by Russo (2007), such a product "[...] is suitable for food and pharmaceutical industries" (Russo, 2007). The 
detailed concentrations of the main polyphenols can be found in Table 3 .

The recent study of Servili et al. (2011) described a RO process using a spiral thin-film membrane made of Durasan $^{\mathrm{TM}}$ and polysulfone for the concentration after pretreatments with MF and UF. The cut-off was determined to be approximately $100 \mathrm{Da}$, the total surface area was $9 \mathrm{~m}^{2}$. The content of total phenols rose from 4.9 $\mathrm{g} \mathrm{L}^{-1}$ (initial OMW)-19.3 $\mathrm{g} \mathrm{L}^{-1}$ in the RO concentrate. Particularly this concentration was made up of 3, 4DHPEA (0.03 $\left.\mathrm{g} \mathrm{L}^{-1}\right)$, p-HPEA (0.01 $\left.\mathrm{g} \mathrm{L}^{-1}\right)$, 3, 4DHPEA-EDA (16.9 $\left.\mathrm{g} \mathrm{L}^{-1}\right)$ and verbascoside $\left(2.4 \mathrm{~g} \mathrm{~L}^{-1}\right)$.

\section{CONCLUSION}

Whereas the environmental impact of olive mill wastewater worsened in the past decades due to industrialization and the involving growth of olive byproducts, the recovery of useful components such as antioxidant phenol compounds from OMW showed great potential to refinance the high costs of the wastewater treatment.

The described studies have proved that membrane separation is an effective technological solution for the ecological problematic olive mill wastewater and involves the recovery of polyphenols and a reduction of the environmental pollution, caused by OMW.

In general, the mentioned studies used MF and/or $\mathrm{UF}$ for the reduction of COD by retaining the suspended solids, which generally contain great parts of the organic substances. Moreover, enzymatic pretreatment was shown as an effective operation to support the COD reduction (Pizzichini and Russo, 2005; Servili et al., 2011). The adjustment of the OMW's pH value was used repeatedly to avoid the oxidation process of the phenolic compounds and therewith guaranteed the quality of the final product. $\mathrm{NF}$ and RO showed their potential for the concentration of the phenolic compounds in the pretreated wastewater. Depending on the initial concentration the final concentrate contained between 0.5 and $30 \mathrm{~g} \mathrm{~L}^{-1}$ total polyphenols. As mentioned by Russo (2007) a concentrate with a polyphenol content of $0.5 \mathrm{~g} \mathrm{~L}^{-1}$ can be used in industry already. Thus, the concentration factor has a greater importance than the final concentration value itself. Furthermore, regarding the results it should be considered, that some experiments used small-scale set-ups and thus are not always significant for real operating conditions.

All in all membrane systems presented sustainable solutions for the pollution problem of OMW and produced polyphenol concentrates, which are valuable for several industry branches.

\section{REFERENCES}

Canepa, P., N. Marignetti, U. Rognoni and S. Calgari, 1988. Olive mills wastewater treatment by combined membrane processes. Water Res., 22: 1491-1494. DOI: 10.1016/0043-1354(88)90160-1

Crittenden, J., 2005. Water Treatment: Principles and Design. 2nd Edn., John Wiley and Sons, Hoboken, N.J., ISBN: 0471110183, pp: 1948.

El-Abbassi, A., A. Hafidi, M.C. Garcia-Payo and M. Khayet, 2009. Concentration of olive mill wastewater by membrane distillation for polyphenols recovery. Desalination, 245: 670-674. DOI: 10.1016/j.desal.2009.02.035

Garcia-Castello, E., A. Cassano, A. Criscuoli, C. Conidi and E. Drioli, 2010. recovery and concentration of polyphenols from olive mill wastewaters by integrated membrane system. Water Res., 44: 3883-3892. DOI: 10.1016/j.watres.2010.05.005

Kapellakis, I.E., K.P. Tsagarakis, C. Avramaki and A.N. Angelakis, 2006. Olive mill wastewater management in river basins: A case study in Greece. Agric. Water Manage., 82: 354-370. DOI: 10.1016/j.agwat.2005.08.004

Marco, E.D., M. Savarese, A. Paduano and R. Sacchi, 2007. Characterization and fractionation of phenolic compounds extracted from olive oil mill wastewaters. Food Chem., 104: 858-867. DOI: 10.1016/j.foodchem.2006.10.005

Niaounakis, M. and C.P. Halvadakis, 2006. Olive Processing Waste Management: Literature Review and Patent Survey. 2nd Edn., Elsevier, Amsterdam, London, ISBN: 0080448518, pp: 498.

Nunes, A.V.M.M., J.P.S.G. Crespo, J.C.D. Santos, A.F. Matias and C.M. Duarte et al., 2007. Method of obtaining a natural hydroxytyrosol-rich concentrate from olive tree residues and subproducts using clean technologies. IP Monitor.

Obied, H.K., M.S. Allen, D.R. Bedgood, P.D. Prenzler and K. Robards et al., 2005. Bioactivity and analysis of biophenols recovered from olive mill waste. J. Agric. Food Chem., 53: 823-837. DOI: 10.1021/jf048569x

Paraskeva, C.A., V.G. Papadakis, E. Tsarouchi, D.G. Kanellopoulou and P.G. Koutsoukos, 2007. Membrane processing for olive mill wastewater fractionation. Desalination, 213: 218-229. DOI: 10.1016/j.desal.2006.04.087

Paraskeva, P. and E. Diamadopoulos, 2006. Technologies for Olive Mill Wastewater (OMW) treatment: A review. J. Chem. Technol. Biotechnol., 81: 1475-1885. DOI: $10.1002 /$ jctb. 1553 
Pizzichini, M. and C. Russo, 2005. Process for recovering the components of olive mill wastewater with membrane technologies. WIPO.

Russo, C., 2007. A new membrane process for the selective fractionation and total recovery of polyphenols, water and organic substances from Vegetation Waters (VW). J. Membrane Sci., 288: 239-246. DOI: 10.1016/j.memsci.2006.11.020

Servili, M., S. Esposto, G. Veneziani, S. Urbani and A. Taticchi et al., 2011. Improvement of bioactive phenol content in virgin olive oil with an olivevegetation water concentrate produced by membrane treatment. Food Chem., 124: 13081315. DOI: 10.1016/j.foodchem.2010.07.042

Stoller, M., 2008. Technical optimization of a dual ultrafiltration and nanofiltration pilot plant in batch operation by means of the critical flux theory: A case study. Chem. Eng. Proces.: Process Intensificat., 47: 1165-1170. DOI: 10.1016/j.cep.2007.07.012
Takac, S. and A. Karakaya, 2009. Recovery of phenolic antioxidants from olive mill wastewater. Recent Patents Chem. Eng., 2: 230-237.

Torrecilla, J.S., 2010. Phenolic compounds in olive oil mill wastewater, olive and olive oil in health and disease prevention. Amsterdam.

Villanova, L., L. Villanova, G. Fasiello and A. Merendino, 2008. Process for the recovery of tyrosol and hydroxytyrosol from oil mill wastewaters and catalytic oxidation method in order to convert tyrosol in hydroxytyrosol. US Patent Application.

Visioli, F., A. Romani, N. Mulinacci, S. Zarini and D. Conte et al., 1999. Antioxidant and other biological activities of olive mill waste waters. J. Agric. Food Chem., 47: 3397-3401. DOI: 10.1021/jf9900534 